\title{
A Rare Case of Helicobacter pylori Infection Complicated by Henoch-Schonlein Purpura in an Adult Patient
}

\author{
Mujtaba Mohamed ${ }^{\mathrm{a}, \mathrm{c}}$, Mohammed Shariffa ${ }^{\mathrm{a}}$, Alsadiq Al Hillan ${ }^{\mathrm{a}}$, Rani Al Haj ${ }^{\mathrm{a}}$, Christian Kaunzinger ${ }^{\mathrm{a}}$, \\ Mohammad Hossain ${ }^{\mathrm{a}}$, Arif Asif ${ }^{\mathrm{a}}$, Nikolaos T. Pyrsopoulos ${ }^{\mathrm{b}}$
}

\begin{abstract}
Henoch-Schonlein purpura (HSP) is known as a leukocytoclastic vasculitis of small vessels, resulting in skin, joint, gastrointestinal (GI) and renal involvement. It is the most common acute vasculitis in children but is relatively uncommon in adults. The pathogenesis of HSP remains unclear, but a wide variety of conditions such as bacterial or viral infections, vaccinations, drugs and other environmental exposures may be responsible for the onset. A few previous case reports have described an association between gastric Helicobacter pylori (HP) infection and HSP. A 30-year-old Indian man who migrated to the USA from India 3 years prior to this presentation with a past medical history of psoriasis on remission, not on any medications presented to the emergency department with sudden onset constant abdominal pain for 5 days. The pain was in the right upper quadrant, spasmodic in nature. The abdominal examination was normal. The patient was evaluated with complete blood count, comprehensive metabolic panel, urinalysis, computed tomography scan of abdomen and pelvis, and right upper quadrant ultrasound, all of which were negative except for leukocytosis. He was discharged on pantoprazole and tramadol. Pain continued despite treatment. Patient was readmitted 2 days later, when he noticed a new maculopapular rash on both legs. Upper GI endoscopy was done showing non-bleeding small gastric ulcer with multiple duodenal erosions. Gastric biopsy came back positive for HP and he was started on clarithromycin, amoxicillin and lansoprazole. Pain persisted after finishing antibiotic course. Rash continued to spread to involve the thighs, flanks, around the umbilicus and extensor surfaces of arms. Immunological workup was negative. A skin biopsy of the skin rash came back positive for leukocytoclastic vasculitis. HSP diagnosis was made by exclusion. Patient was started on prednisone $40 \mathrm{mg}$ daily and improved drastically on the following day. He was continued steroids taper for 8 weeks. Rash as well as
\end{abstract}

Manuscript submitted April 27, 2020, accepted May 7, 2020

Published online May 28, 2020

aDepartment of Medicine, Jersey Shore University Medical Center, Hackensack Meridian Health, Neptune, NJ 07753, USA

${ }^{\mathrm{b}}$ Division of Gastroenterology and Hepatology, Department of Medicine, Rutgers New Jersey Medical School, 185 South Orange Avenue, H-532, Newark, NJ 07103, USA

${ }^{\mathrm{c}}$ Corresponding Author: Mujtaba Mohamed, Department of Medicine, Jersey Shore University Medical Center, Hackensack Meridian Health, Neptune, NJ 07753, USA. Email: mujtaba.mohamed@hackensackmeridian.org

doi: https://doi.org/10.14740/jmc3480 abdominal pain resolved completely. In conclusion, HSP involves the skin, GI tract, joints and kidneys. It is a pediatric disease and rarely occurs in adults. It can be associated with underlying malignancy in adults. HP infection can trigger HSP in pediatric and adult patients. Detection of the carrier state is crucial in HSP patients in areas where HP is endemic. Eradication of HP infection is usually associated with the resolution of HSP. In resistant cases with GI and renal involvement, corticosteroids use results in resolution of symptoms and reduction of the duration of mild nephritis.

Keywords: Helicobacter pylori; Henoch-Schonlein purpura; Abdominal pain

\section{Introduction}

Henoch-Schonlein purpura (HSP) is a leukocytoclastic vasculitis of small vessels with deposition of IgA, commonly resulting in skin, joint, gastrointestinal (GI) and kidney involvement. The first description was attributed to Heberden in 1806 , but Schonlein first described the association of arthralgias and purpura, termed "peliosis rheumatica", in 1837. His student, Henoch, described the GI manifestations in 1874 and renal involvement in 1899 [1]. It is mainly a pediatric disease. Typical presentation in children includes abdominal pain and a vasculitic purpuric rash, primarily over the lower extremities, sometimes accompanied by hematuria and arthralgias or arthritis. It is a self-limited disease, often resolving within a month, particularly if no renal involvement is demonstrated [2]. The possible relation between Helicobacter pylori (HP) infection and HSP was firstly enlightened by several case reports in adults suffering HSP and gastritis simultaneously [3-5]. The HP infection could be detected by the 13C-urea breath test. The standard diagnostic test relies on gastric mucosal biopsy. Herein we present a rare case report of an Indian adult man diagnosed with HP infection complicated by HSP.

\section{Case Report}

A 30-year-old Indian man who migrated to the USA from India 3 years prior to this presentation with a past medical history of psoriasis, not on any medications presented to the emergency 


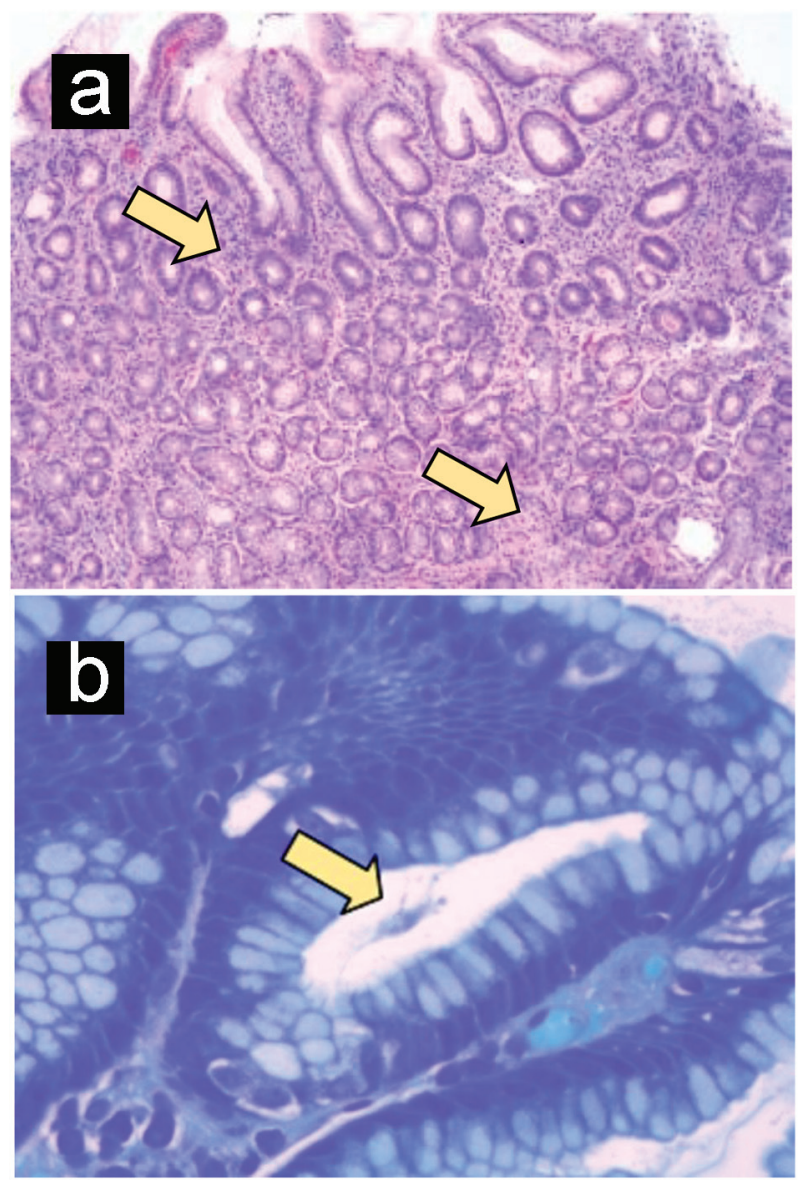

Figure 1. Helicobacter pylori gastritis. (a) Gastric mucosa shows intense chronic inflammation. (b) Giemsa stain identifies curved, spirochete-like Helicobacter pylori organisms (arrow). Original magnification, $\times 100(\mathrm{a}) ; \times 630(\mathrm{~b})$.

department (ED) with sudden onset constant abdominal pain for 5 days. The pain was in the right and left upper quadrant, spasmodic in nature, 5/10 in intensity, not radiating and no aggravating or relieving factors. The abdominal examination did not reveal any guarding, or any other abnormality. In the ED, he was evaluated with a complete blood count, metabolic panel, urinalysis, computed tomography (CT) abdomen and pelvis, and right upper quadrant ultrasound, all of which were negative except for leukocytosis $17.9\left(4.5-11 \times 10^{3} / \mu \mathrm{L}\right)$. He was discharged from the ED with a prescription for pantoprazole and tramadol.

The patient returned to the ED 2 days later with persistent abdominal pain associated with nausea, vomiting and inability to tolerate an oral diet. At this time, he also reported maculopapular rash that he noticed on both legs which started at the same time with abdominal pain. He reported rash extended to his calves. He was admitted for further evaluation and underwent endoscopy which showed non-bleeding small gastric ulcer with multiple duodenal erosions (Fig. 1a). He was treated symptomatically and discharged on pantoprazole. On day 12 (since the onset of pain), the gastric biopsy came back positive for HP (Fig. 1b) and he was started on triple therapy (clarithromycin, amoxicillin and lansoprazole) for HP.

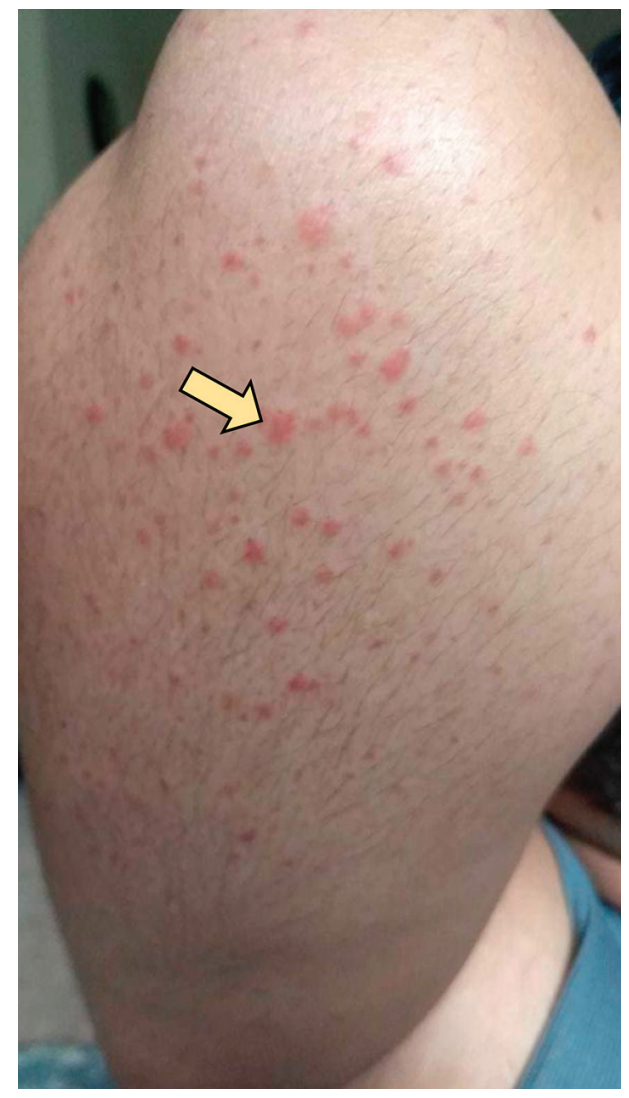

Figure 2. Maculopapular rash around his right elbow.

The patient completed the 2 weeks of triple therapy; however, abdominal pain persisted. The rash continued to spread to involve the thighs, flanks, around the umbilicus, and extensor surfaces of arms (Figs. 2, 3). The patient came back to the ED on day 27 (since the onset of symptoms) with the same symptoms after finishing triple antibiotic therapy. The patient was again admitted and underwent extensive immunological workup including rheumatoid factor, C-reactive proteins, anti-nuclear antibodies, total complement level, C3, C4, IgA level, ANCA IgG level, myeloperoxidase antibody, serine protein 3, anti-DNA antibody and erythrocyte sedimentation rate, which were all within normal limits. He underwent repeat endoscopy which showed healed gastric ulcer. Skin rash continued to worsen to involve almost all extensor surfaces of both legs (Fig. 4). A skin biopsy of the skin rash was obtained while the patient was treated symptomatically with narcotics to control the pain. The patient was eventually discharged with pain medications and instructed to follow-up as an outpatient. The skin biopsy eventually came back positive for leukocytoclastic vasculitis (Fig. 5). HSP diagnosis was made by exclusion since patient did not have connective tissue disorders based on immunological workup, no history of drug ingested prior to symptoms. At that point, the patient was started on prednisone $40 \mathrm{mg}$ daily and improved drastically on the following day of starting steroids. Patient continued on steroids taper for 8 weeks. Rash as well as abdominal pain resolved completely. 


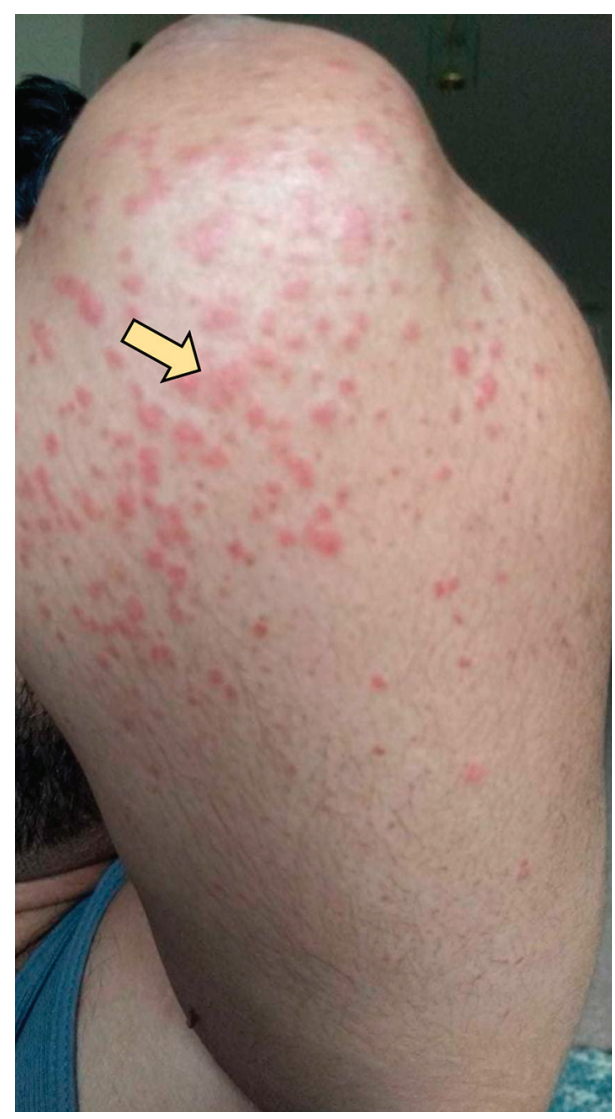

Figure 3. Maculopapular rash around his left elbow.

\section{Discussion}

HSP is known as a leukocytoclastic vasculitis of small vessels, resulting in skin, joint, GI and renal involvement. It is the most common acute vasculitis disorder affecting children; however, it is relatively uncommon in adults [6]. Prior to the last 30 years, it was considered a disease of children only, with an estimated incidence of 20 cases/100,000 children per year [2]. It recurs in approximately one-third of children. An upper respiratory infection is the primary precipitating event in children, although approximately two-thirds of children with HSP have no precipitating event [2]. This disorder is encountered even more rarely by nephrologists caring for adults and accounts for $0.6-2 \%$ of adult nephropathies [6]. HSP in adults can initially present as malignant lung, breast, prostate and intestine carcinomas (66\%) and 33\% are hematological such as lymphoma and multiple myeloma [7]. Reinauer et al [3] reported the first HSP and chronic gastritis case with diagnosed HP infection by $13 \mathrm{C}$-urea breath test in 1995, in whom the purpura, intestinal symptoms and albuminuria disappeared after eradication treatment. A subsequent case was reported by Mozrzymas et al [8]. Mytinger et al [9] reported HSP induced by HP in pediatric patients. Multiple articles in China reported a potential association between HP infection and HSP [10]. Thus, detecting HP carrying status in HSP children, particularly those with abdominal manifestations, is indispensable in endemic areas.

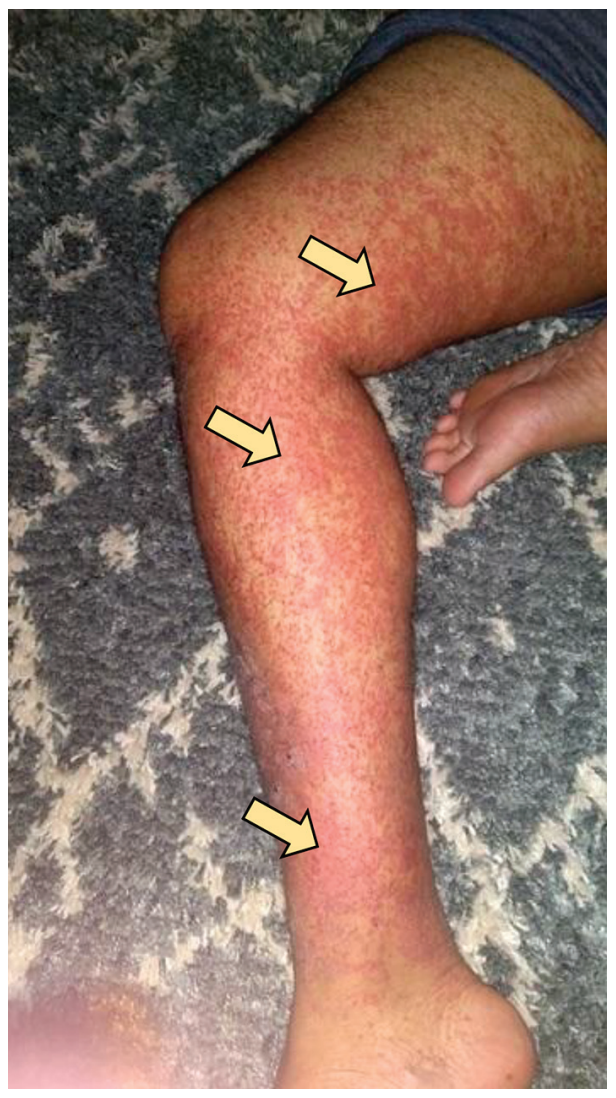

Figure 4. Skin rash continued to worsen to involve almost all extensor surfaces of both thigs and legs.

Diagnostic methods that can confirm the current infection situation are recommended to detect the existence of HP in HSP patients. Our patient was diagnosed by gastric biopsy.

$\mathrm{HP}$ is a known etiology for gastric diseases such as gastritis [11], peptic ulcer [12], gastric cancer and B-cell lymphoma of gastric mucosa-associated-lymphoid-tissue (MALT lymphoma) [13]. In addition to local tissue damage, a potential role of HP infection in various extragastric pathologies has been described [14]. Autoimmune diseases include HSP, Sjogren's syndrome, autoimmune thrombocytopenia, acute immune polyneuropathies [15]. Association with other diseases such as ischemic heart disease and membranous nephropathy was described as well [15].

The mechanism of HSP and HP is unclear. The presence of more toxic HP strains induces a strong inflammatory response, directly or indirectly, with the release of bacterial and hostdependent cytotoxic substances [15], genetic predisposition of the individual [14], antibodies against HP cross-reaction with some extragastric tissues [16], polymeric immunoglobulin A (pIgA), activated complements (C3 or C5) and certain fibrinogen/fibrin deposited in vessel walls, without IgG or IgM deposition. The immune complex between these elements in skin, gut, kidney and other organs resulted in purpura, intestinal manifestation, nephritis and other relatively rare symptoms. Most investigators agreed that IgA1 was crucial in the progression of HSP $[17,18]$. It is most likely the pathogen 

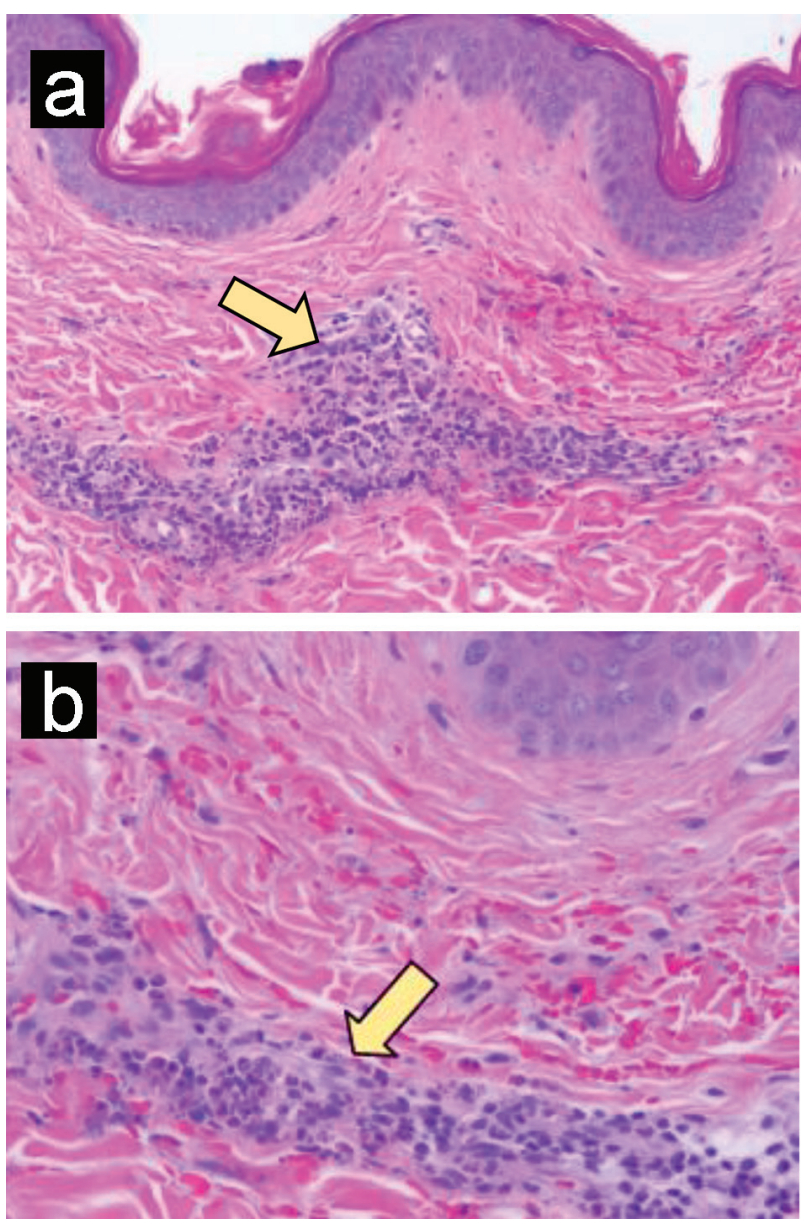

Figure 5. Leukocytoclastic vasculitis. (a) Skin biopsy shows perivascular inflammatory cell infiltrate and erythrocyte extravasation. (b) Perivascular neutrophilic infiltrate with karyorrhexis (arrow). Original magnification, $\times 200(a) ; \times 400(b)$.

raises IgA levels resulting in type III hypersensitivity allergic reaction. Another factor is virulence of HP. The products of vacuolating toxin gene $\mathrm{A}(\mathrm{vacA})$ and cagA are the main virulence factors of HP. The vacA and cagA alleles encoded the most important HP virulence proteins VacA and CagA, which has a high prevalence in China. Based on the high toxigenicity of Chinese HP strains and its association with HSP [18] and relatively low toxigenicity of strains in western countries, it is though that vacA or cagA might participate in the progression of HSP through a complicated and unknown mechanism [19].

Diagnosis is confirmed by the presence of IgA by immunofluorescence in purpuric skin lesions or IgA and C3 complement mesangial deposits on kidney biopsy. In our patient, diagnosis was made by skin right arm punched biopsy which showed leukocytoclastic vasculitis; however, IgA staining was not done.

Mytinger et al [9] reported a 13-year-old boy with HSP associated with HP infection who was treated with triple therapy antibiotics and prompt resolution of the HSP was achieved. However, in this patient, abdominal pain and rash persisted even after completion of triple antibiotic therapy and resolu- tion of HP infection.

In general, patients with HSP are treated supportively or symptomatically, whereas in patients with severe GI manifestations or renal complications, corticosteroid therapy is considered. Recently, a randomized controlled study confirmed that corticosteroid therapy is effective in reducing the intensity of abdominal pain and joint pain and in shortening the duration of mild nephritis [20]. However, there is no evidence that it is effective in preventing recurrence or the development of nephritis. Dapsone is believed by some to have beneficial effects on cutaneous, GI and joint manifestations [21]. Pulse methylprednisolone therapy and such immunosuppressive agents as azathioprine and cyclophosphamide have been used anecdotally for severe kidney disease or another life-threatening disease. There are case reports of the efficacy of cyclosporine [22], intravenous immunoglobulin [23], plasmapheresis [24] and thalidomide [25] in patients that were resistant to steroids. In our patient, HSP symptoms persisted even after completing the triple therapy antibiotic course. Abdominal pain resolved after the initiation of the steroids course.

Prognosis of the typical course of kidney disease associated with adult HSP progresses over months, and a minority of patients will present with rapidly progressive glomerulonephritis (RPGN). The literature demonstrates multiple case reports of acute kidney failure in adults due to $\operatorname{HSP}[26,27]$. Thirty-eight percent of adult patients with HSP demonstrated kidney disease. Of these, $85 \%$ had microhematuria, $10 \%$ had macroscopic hematuria and 32\% had impaired renal functions. End-stage kidney disease was reached in $11 \%$ of patients, and half of these occurred in less than 3 years from the onset of the disease. Factors predictive of the poor renal outcome included advanced age, preexisting chronic kidney disease, proteinuria more than $1 \mathrm{~g}$ /day, macroscopic hematuria, advanced pathological stage of kidney disease and glomerular sclerosis and interstitial fibrosis on kidney biopsy [27, 28].

\section{Conclusion}

HSP, also known as a leukocytoclastic vasculitis of small vessels, primarily involves the skin, GI tract, joints and kidneys. It is a pediatric disease; however, it can occur in adults. It can be associated with underlying malignancy in adults. HP infection can trigger HSP in pediatric and adult patients. Detection of the carrier state is crucial in HSP patients in areas where HP is endemic. Eradication of HP infection is usually associated with the resolution of HSP. In resistant cases with GI and renal involvement, corticosteroids use results in resolution of symptoms and reduction of the duration of mild nephritis.

\section{Acknowledgments}

We would like to thank Chunwei, Ma (Liberian at Jersey Shore University Medical Center) for the help of providing us with other case reports of HSP in adults to compare them to our case. We would also like to thank Dr. Min Zheng, MD, PhD 
for providing us with the pathology slides for gastric and skin biopsy.

\section{Financial Disclosure}

Author has no financial interest to disclose.

\section{Conflict of Interest}

The authors declare that there is no conflict of interest regarding the publication of this paper.

\section{Informed Consent}

Written informed consent was obtained from the patient for publication of this case report and any accompanying images.

\section{Author Contributions}

Each author has individually been involved and participated in drafting the manuscript and revising it critically for important intellectual content and have given final approval of the version to be published. Each has agreed to be accountable for all aspects of the work in ensuring that questions related to the accuracy or integrity of any part of the work are appropriately investigated and resolved. NP, AA, MH and CK encouraged MM, MS and AA to learn about HSP in adults and its clinical presentation. All authors discussed the medical literature. MM presented the idea. AA and MM wrote the manuscript with input from all authors.

\section{Data Availability}

The authors declare that data supporting the findings of this study are available within the article.

\section{References}

1. Rai A, Nast C, Adler S. Henoch-Schonlein purpura nephritis. J Am Soc Nephrol. 1999;10(12):2637-2644.

2. Coppo R, Mazzucco G, Cagnoli L, Lupo A, Schena FP. Long-term prognosis of Henoch-Schonlein nephritis in adults and children. Italian Group of Renal Immunopathology Collaborative Study on Henoch-Schonlein purpura. Nephrol Dial Transplant. 1997;12(11):2277-2283.

3. Reinauer S, Megahed M, Goerz G, Ruzicka T, Borchard F, Susanto F, Reinauer H. Schonlein-Henoch purpura associated with gastric Helicobacter pylori infection. J Am Acad Dermatol. 1995;33(5 Pt 2):876-879.

4. Machet L, Vaillant L, Machet MC, Buchler M, Lorette G. Schonlein-Henoch purpura associated with gastric Helicobacter pylori infection. Dermatology. 1997;194(1):86.
5. Cecchi R, Torelli E. Schonlein-Henoch purpura in association with duodenal ulcer and gastric Helicobacter pylori infection. J Dermatol. 1998;25(7):482-484.

6. Blanco R, Martinez-Taboada VM, Rodriguez-Valverde V, Garcia-Fuentes M, Gonzalez-Gay MA. Henoch-Schonlein purpura in adulthood and childhood: two different expressions of the same syndrome. Arthritis Rheum. 1997;40(5):859-864.

7. Pertuiset E, Liote F, Launay-Russ E, Kemiche F, CerfPayrastre I, Chesneau AM. Adult Henoch-Schonlein purpura associated with malignancy. Semin Arthritis Rheum. 2000;29(6):360-367.

8. Mozrzymas R, d'Amore ES, Montini G, Guariso G. Schonlein-Henoch vasculitis and chronic Helicobacter pylori associated gastritis and duodenal ulcer: a case report. Pediatr Med Chir. 1997;19(6):467-468.

9. Mytinger JR, Patterson JW, Thibault ES, Webb J, Saulsbury FT. Henoch-Schonlein purpura associated with Helicobacter pylori infection in a child. Pediatr Dermatol. 2008;25(6):630-632.

10. Wang YL, Xue YZ. Explore the relationship between Helicobacter pylori infection and Henoch-Schonlein purpura. Sichuan Yixue. 2004;25:176-177.

11. Buck GE, Gourley WK, Lee WK, Subramanyam K, Latimer JM, DiNuzzo AR. Relation of Campylobacter pyloridis to gastritis and peptic ulcer. J Infect Dis. 1986;153(4):664-669.

12. Graham DY. Campylobacter pylori and peptic ulcer disease. Gastroenterology. 1989;96(2 Pt 2 Suppl):615-625.

13. Kelly SM, Geraghty JM, Neale G. H pylori, gastric carcinoma, and MALT lymphoma. Lancet. 1994;343(8894):418.

14. Realdi G, Dore MP, Fastame L. Extradigestive manifestations of Helicobacter pylori infection: fact and fiction. Dig Dis Sci. 1999;44(2):229-236.

15. Gasbarrini A, Franceschi F. Autoimmune diseases and Helicobacter pylori infection. Biomed Pharmacother. 1999;53(5-6):223-226.

16. Ko GH, Park HB, Shin MK, Park CK, Lee JH, Youn HS, Cho MJ, et al. Monoclonal antibodies against Helicobacter pylori cross-react with human tissue. Helicobacter. 1997;2(4):210-215.

17. Davin JC, Ten Berge IJ, Weening JJ. What is the difference between IgA nephropathy and Henoch-Schonlein purpura nephritis? Kidney Int. 2001;59(3):823-834.

18. Touchard G, Maire P, Beauchant M, Doeuvre P, Babin P, Pecheur H, Becq-Giraudon B, et al. Vascular IgA and C3 deposition in gastrointestinal tract of patients with Henoch-Schoenlein purpura. Lancet. 1983;1(8327):771772.

19. Franceschi F, Sepulveda AR, Gasbarrini A, Pola P, Silveri NG, Gasbarrini G, Graham DY, et al. Cross-reactivity of anti-CagA antibodies with vascular wall antigens: possible pathogenic link between Helicobacter pylori infection and atherosclerosis. Circulation. 2002;106(4):430-434.

20. Ronkainen J, Koskimies O, Ala-Houhala M, Antikainen M, Merenmies J, Rajantie J, Ormala T, et al. Early prednisone therapy in Henoch-Schonlein purpura: a randomized, double-blind, placebo-controlled trial. J Pediatr. 2006;149(2):241-247. 
21. Shimomura N, Kawai K, Watanabe S, Katsuumi K, Ito M. Adult Henoch-Schonlein purpura with severe abdominal pain treated with dapsone and factor XIII concentrate. J Dermatol. 2005;32(2):124-127.

22. Harries MJ, McWhinney P, Melsom R. Recurrent Henoch-Schonlein purpura controlled with ciclosporin. J R Soc Med. 2004;97(4):184-185.

23. Kusuda A, Migita K, Tsuboi M, Degawa M, Matsuoka N, Tominaga M, Kawakami A, et al. Successful treatment of adult-onset Henoch-Schonlein purpura nephritis with high-dose immunoglobulins. Intern Med. 1999;38(4):376-379.

24. Chen TC, Chung FR, Lee CH, Huang SC, Chen JB, Hsu KT. Successful treatment of crescentic glomerulonephritis associated with adult-onset Henoch-Schoenlein purpura by double-filtration plasmapheresis. Clin Nephrol.
2004;61(3):213-216.

25. Choi SJ, Park SK, Uhm WS, Hong DS, Park HS, Park YL, Kwon KW. A case of refractory Henoch-Schonlein purpura treated with thalidomide. Korean J Intern Med. 2002;17(4):270-273.

26. Kassutto S, Wolf MA. Clinical problem-solving. A wrinkle in time. N Engl J Med. 2003;349(6):597-601.

27. Pillebout E, Thervet E, Hill G, Alberti C, Vanhille P, Nochy D. Henoch-Schonlein Purpura in adults: outcome and prognostic factors. J Am Soc Nephrol. 2002;13(5):12711278.

28. Tancrede-Bohin E, Ochonisky S, Vignon-Pennamen MD, Flageul B, Morel P, Rybojad M. Schonlein-Henoch purpura in adult patients. Predictive factors for IgA glomerulonephritis in a retrospective study of 57 cases. Arch Dermatol. 1997;133(4):438-442. 